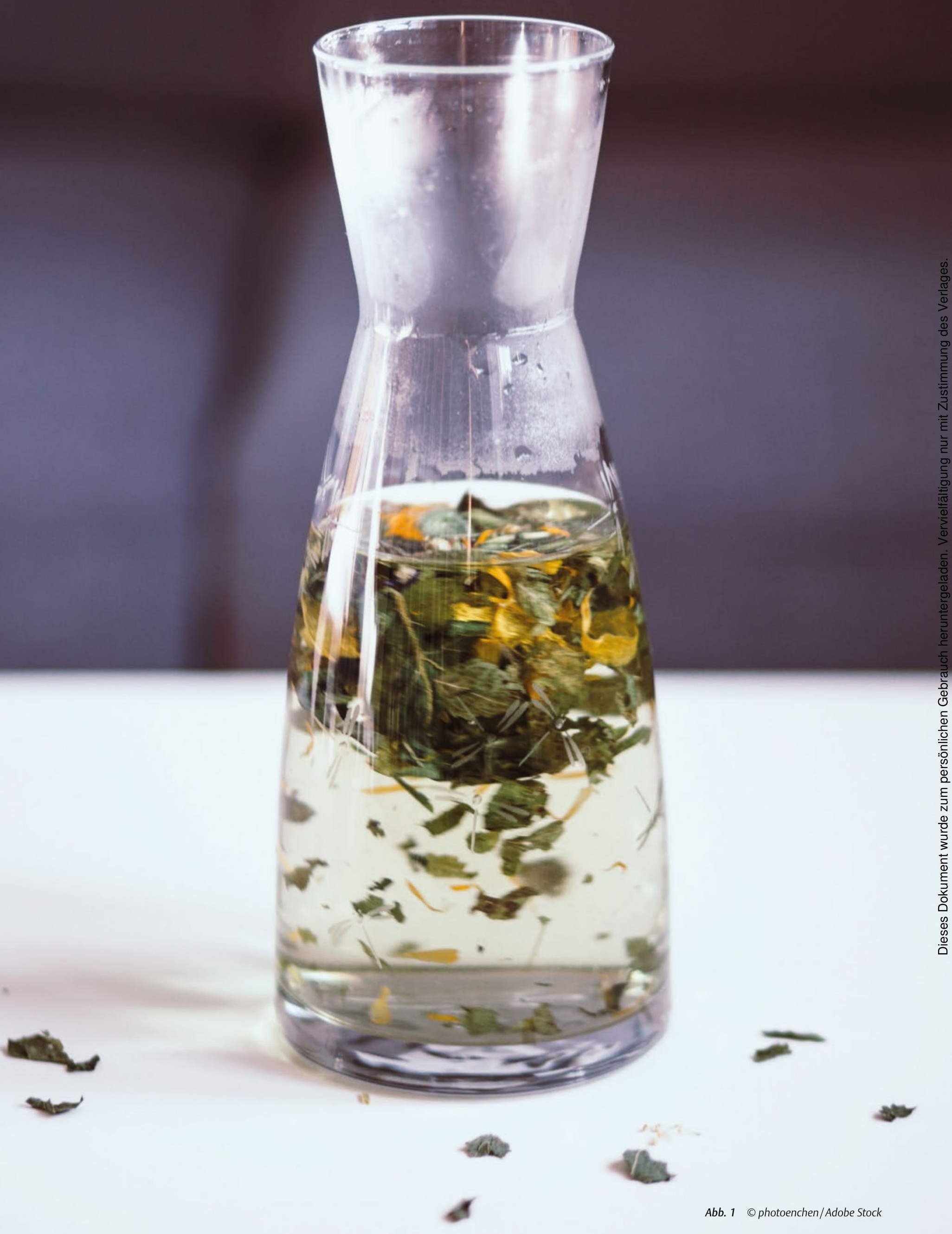




\section{Aufbruch in der}

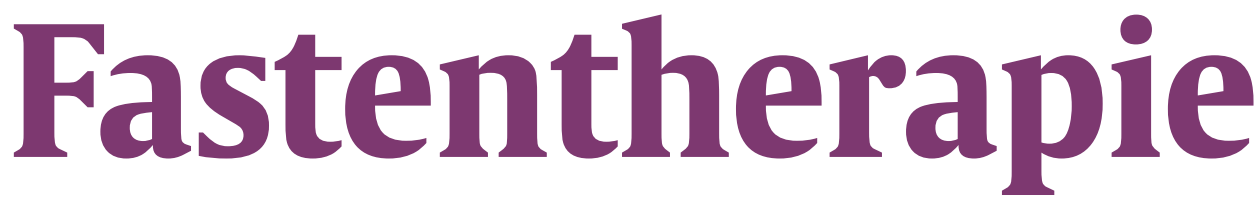

Vom Heilfasten zum Intervallfasten, Scheinfasten und periodischen Fasten - Ein Überblick

Andreas Michalsen

\section{Einführung}

Zeiträume bewussten Fastens mit Beschränkung der Aufnahme fester Nahrung werden weltweit praktiziert und sind inzwischen fester Bestandteil der europäischen klassischen Naturheilkunde in Form des Heilfastens oder therapeutischen modifizierten Fastens.

Die neuere Geschichte des medizinischen Fastens basiert wesentlich auf den Arbeiten und dem Wirken der Ärzte Otto Buchinger, Herbert Krauß sowie Franz Xaver Mayr. Buchinger, selbst geprägt von einer heilenden Fastenerfahrung in der Klinik von Dr. Riedlin, begründete das Konzept des modifizierten therapeutischen Fastens („Heilfasten“) im Gegensatz zur Nulldiät, dem Wasserfasten, das schon Jahrzehnte zuvor in den USA starke Verbreitung bei Ärzten der „natural hygiene“ fand.

\section{Zusammenfassung}

Alternierende Phasen des Nahrungsüberangebots und des Hungerns waren bei den Frühmenschen vermutlich der Regelfall. Es scheint naheliegend, dass der menschliche Körper evolutionsbedingt Phasen des Fastens sehr gut bewältigen kann und sich die genetische Ausstattung, der Körpermetabolismus und die Ernährungsphysiologie gut darauf eingestellt haben. Im Gegensatz dazu ist anzunehmen, dass die mehrmals tägliche, regelmäßige Nahrungszufuhr mit Snacks, Zwischenmahlzeiten und energiehaltigen Getränken, die Hormon- und Stoffwechselsysteme überfordert.

Die günstigen metabolischen Wirkungen des Fastens sind in physiologischen Arbeiten beschrieben. Der Artikel gibt einen Überblick zu den verschiedenen Formen des Fastens und dessen mögliche Effekte.

\section{Modifiziertes Fasten nach Buchinger}

Beim modifizierten Fasten nach Buchinger wird eine gewisse Kalorienmenge dem Körper in Form von definierter flüssiger Nahrung täglich zugeführt. Diese Kalorienmenge soll nach dem damaligen Konzept 500 kcal nicht überschreiten, um die fasteninduzierte hepatisch gesteuerte Lipolyse nicht zu hemmen. Allerdings ist die Kalorienobergrenze dieser Grenze physiologisch nicht genau determiniert. Auf eine Nulldiät wird im Gegensatz zur Tradition des amerikanischen Fastens verzichtet, um einen verstärkten Proteinkatabolismus und konsekutiven Muskelabbau zu vermeiden. Schließlich wird auf feste Nahrung verzichtet, um Hunger, der möglicherweise durch Kauen induziert wird, nicht zu fördern. Diese Form des modifizierten Fastens nach Buchinger ist die derzeit in Europa am häufigsten eingesetzte Fastenmethode und wird meist auch als Saftfasten bezeichnet.

Weitere Fastenformen, die klinisch und ambulant eingesetzt werden, sind das sog. Schleimfasten (Reis- oder Getreideschleim), insbesondere bei magenempfindlichen Menschen bzw. vorliegenden Magenerkrankungen. Ein weiteres wesentliches Charakteristikum des therapeutischen Fastens bzw. Heilfastens ist, dass es sich um ein multimodales Therapieprogramm handelt, also die Therapiemethode nicht nur die Beschränkung der Kalorienzufuhr umfasst, sondern in ein therapeutisches ganzheitliches Therapieprogramm eingebettet ist. Im Konzept von Buchinger, aber auch anderer etablierter Verfahren, wird besonderer Wert auf Bewegungstherapie, aber auch Entspannungsverfahren gelegt. Zusätzlich wird die sog. Ausleitung über das Anregen des Schwitzens (Diaphorese), der Diurese (reichlich Trinken) sowie der Purgation (abführende Salze, Einläufe) unterstützt. Darüber hinaus gibt es standardisierte, begleitende Therapien wie den täglichen Leberwickel sowie Schulungen zu gesunder Ernährung und einem allgemein gesundheitsfördernden Lebensstil. Dieses Gesamtkonzept ist eindeutig zu favorisieren gegenüber reinen, auf die Kalorienreduktion fokussierten Diät- oder Fastenformen.

\section{Periodisches Fasten}

Inzwischen wurde von den amerikanischen Wissenschaftlern und Fastenexperten Longo und Mattson vorgeschlagen, das Fasten über längere Zeiträume von Tagen und Wochen als periodisches Fasten zu bezeichnen. Das periodische Fasten für Gesunde dauert hierbei in der Regel 5 oder 7 Tage. Das Heilfasten bzw. therapeutische Fasten hat meist eine Therapiedauer von 7, 10 oder 14 Tagen, kann aber bei ausreichendem Ausgangsgewicht oder Übergewicht bis zu 4 oder sogar 6 Wochen ausgeübt werden.

Als eine modifizierte Form des periodischen Fastens ist die „fasting mimicking diet“ (FMD), das „Scheinfasten“ nach Valter Longo anzusehen [11]. Hierbei stehen 2 Formen, die inzwischen als Produkt vermarktet werden, zur Verfügung.

1. Die „fasting mimicking diet“ während der Chemotherapie („Chemolieve“ 96 Stunden adaptiert auf Geschmacksempfindlichkeiten von Patienten mit Krebserkrankungen) sowie

2. die konventionelle fasting mimicking diet („Prolon“), die empfohlen wird, monatlich an 4-5 Tagen einzusetzen. Die Kalorienzufuhr beträgt hierbei 7001000 kcal täglich. 
Es bleibt jedoch abzuwarten, ob sich diese Form des Fastens durchsetzt, da sie mit einem erheblichen Kostenmehraufwand gegenüber dem natürlichen Fasten verbunden ist.

Neben diesem periodischen, prolongierten Fasten bzw. Heilfasten, wurde in den letzten Jahren das intermittierende Fasten oder Intervallfasten äußerst populär. Darunter werden kürzere Fastenphasen oder aber auch einzelne Fastentage in definierten Rhythmen, wie 5:2-Fasten, 1:6-Fasten oder „10in2“ (10 Stunden in 2 Tagen) verstanden. Interessanterweise ist das intermittierende Fasten, im Gegensatz zum durch Erfahrung entstandenen Heilfasten, eine Konsequenz der Translation tierexperimenteller Studien, die über Jahrzehnte konsistent belegten, dass eine kalorische Restriktion um 20-30\% oder eben zeitlich begrenzte intermittierende Fütterungsperioden im Gegensatz zu „ad libitum“-Füttern/Essen bei allen biologischen Organismen zu einer Lebensverlängerung und Verschiebung altersassoziierter chronischer Erkrankungen führen. Im Folgenden werden die aus der Forschung abgeleiteten Fastenformen näher beschrieben.

\section{Kontinuierliche Kalorische Restrik-} tion (Calorie restriction, CR)

Zurückgehend auf den Grundlagenwissenschaftler und Pathologen Roy Walford (Initiator der Biosphere) wurde initial versucht, das Konzept der dauerhaften kalorischen Restriktion auf den Menschen zu übertragen. Noch heute gibt es in den USA die sog. CRONies (Caloric restriction on optimum nutrition) die dies praktizieren. Allerdings entstehen bei der CR erhebliche Anforderungen an die Compliance und einige Nachteile der dauerhaften kalorischen Restriktion wie Untergewicht, Frieren, sexuelles Desinteresse und Infektanfälligkeit, die sie für die Breitenanwendung ziemlich ungeeignet erscheinen lässt. handen. In der Regel kommt es zu keinem Untergewicht, sondern nur zur Gewichtsabnahme bis zur Gewichtsnormalisierung.

\section{Die 2-Tage-Diät}

2013 hat die Wissenschaftlerin Dr. Michelle Harvie aus Machester, UK, zusammen mit dem Onkologen Tony Howell das Konzept der „2-day-diet“ entwickelt, um die Gewichtsabnahme zu erleichtern [12]. Diese Diätform wurde ursprünglich für Brustkrebspatientinnen entwickelt. Es werden an 2 aufeinanderfolgenden Tagen in der Woche jeweils höchstes $600 \mathrm{kcal}$ an Nahrungsenergie verzehrt. Es sollte kohlenhydratarm gegessen werden. Sie empfiehlt v.a. Milchprodukte, Tofu, Gemüse, Fisch, Obst und Eier. An den anderen Tagen sollte man mediterran essen.

\section{5:2-Fasten}

Diese Fastenform hat der Arzt und Wissenschaftsjournalist Michael Moseley auf Grundlage des Konzeptes von Harvie definiert und durch einen Bestseller (,the fast diet“) stark befeuert [13]. 5:2 heißt dabei, an 2 Tagen, die nicht aufeinanderfolgen, werden nur ca. 600 kcal gegessen, meist auf 2 Mahlzeiten mit je $300 \mathrm{kcal}$ verteilt. Dadurch, dass es nicht 2 aufeinanderfolgende Tage sind, fällt es den meisten Menschen leichter, das Fasten beizubehalten. Moseley empfiehlt v.a. Gemüse und Vollkorngetreide sowie reichlich Flüssigkeit an den Fastentagen. Michelle Harvie hat die meisten klinischen Studien zum 5:2-Fasten durchgeführt. Hierbei war diese Form des intermittierenden Fastens einer kontinuierlichen kalorischen Restriktion hinsichtlich der Gewichtsabnahme ebenbürtig, dabei aber durch bessere Compliance und stärkere Reduktion der Fettmasse charakterisiert.

\section{Intermittierendes Fasten verursacht i.d. R. kein Un-} tergewicht, sondern führt zur Gewichtsabnahme

\section{bis zur Gewichtsnormalisierung.}

\section{Intermittierendes Fasten}

Die Nachteile der CR sind interessanterweise beim intermittierenden Fasten nicht vor-

\section{Alternate day diet}

Die Alternate day diet (AD) firmiert auch unter dem Begriff „eat stop eat“, „upday- downday“. Das Konzept wurde erstmals von Christa Varady 2013 in ihrem Buch the „every other day diet“" veröffentlicht. An den Fastentagen isst man dabei nur $25 \%$ der vollen Menge, am darauffolgenden Tag so viel wie man möchte. Mehrere kleinere klinische Studien zur AD wurden durchgeführt. Hierbei zeigte sich eine ähnliche Gewichtsabnahme wie bei täglicher kalorischer Restriktion mit leichten Vorteilen für AD hinsichtlich des Insulinspiegels und der Reduktion der Fettmasse. Einschränkend muss aber festgestellt werden, dass es den meisten Menschen nicht leicht fällt in einem zweitägigen Rhythmus kontinuierlich zu fasten und dies auch sozial Nachteile mit sich bringen kann. In einem laufenden Forschungsprojekt der Universität Graz (InterFAST-Studie) wurde andererseits das ADFasten erfolgreich implementiert [14]. Das Forscherteam um Frank Madeo geht davon aus, dass die Autophagie maßgeblich durch Intervallfasten gesteigert wird [15].

Eine spezielle Form der $A D$ ist das „10in2“ (das der bekannte Wiener Kabarettist Bernhard Ludwig populär gemacht hat). Hierbei wird nur jeden 2. Tag und dann alles innerhalb 10 Stunden gegessen, sodass regelmäßig eine Pause von 38 Stunden entsteht.

\section{Insbesondere bei Diabe-}

\section{tes mellitus sind ein-}

\section{drückliche günstige}

\section{Wirkungen für 16:8}

\section{beschrieben.}

\section{Time restricted feeding/Time restricted} eating (TRF/TRE)

Besonders attraktiv erscheint die Modulation der Tagesrhythmik des Essens. Im angloamerikanischen Raum hat sich hierfür der Begriff time restricted feeding durchgesetzt. Unklar ist aber bislang, auch wenn immer mehr populäre Bücher schon von Beweisen sprechen, welches Intervall von regelmäßigen Fastenperioden das Maximum an therapeutischem Benefit mit sich bringt. Beim time restricted feeding ist davon auszugehen, dass eine tägliche Essenspause von 1416 Stunden oder max. 18 Stunden das Opti- 
Fasten Praxis zkm

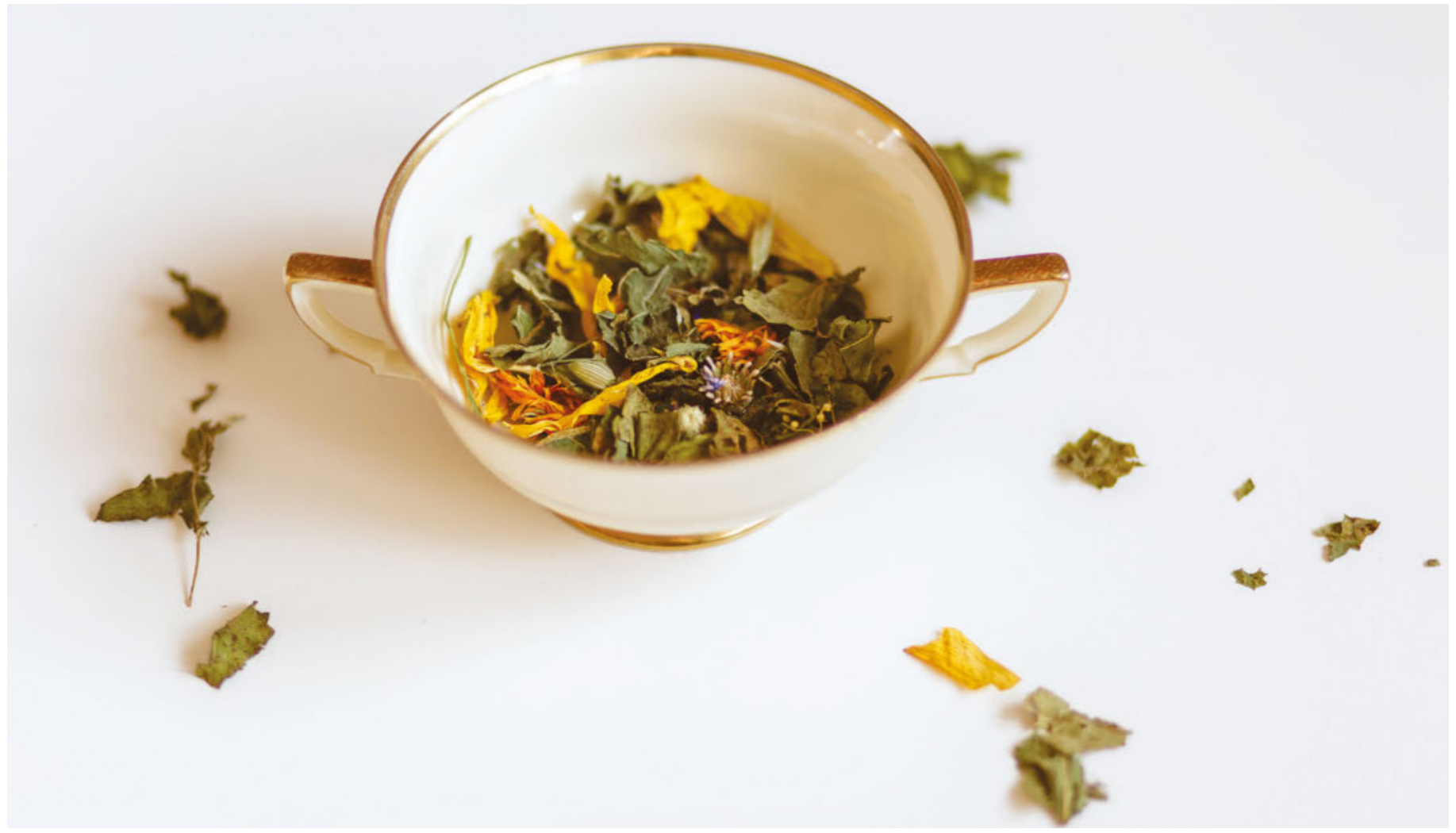

Abb. 2 @ photoenchen/Adobe Stock

mum darstellt. Möglicherweise sind die Glykogenreserven der Leber für den Effekt entscheidend. Bei Frauen sind diese Glykogenvorräte vermutlich etwas weniger lang ausreichend (14 Stunden), bei Männern vermutlich 16-18 Stunden. Entsprechend beginnt bereits nach 12-14 Stunden Fasten der erste Anstieg der Ketonkörperproduktion im Körper.
In der praktischen Umsetzung kann Patienten empfohlen werden, das natürliche Nachtfasten zu verlängern, indem entweder das Frühstück ausgelassen (breakfast fasting; „lean gains“) oder verspätet eingenommen wird, oder das Abendessen weggelassen („dinner cancelling“) oder früher eingenommen wird. Dies Form des Interfallfastens ist i.d.R. leicht praktikabel. Wichtig erscheint auch, dass die Gesamtkalorienmenge nicht bewusst verändert werden muss. Klinische Studien zeigen aber, dass allein durch die Reduktion der Mahlzeitenfrequenz auf 2 pro Tag i.d.R. bei den meisten Menschen eine Reduktion der Energiezufuhr und konsekutiv eine Gewichtsabnahme entsteht.

Die Entscheidung, ob die Nachtfastenperiode nach hinten oder vorn verlängert wird, 
sollte individuell nach chronobiologischen individuellen Aspekten und den sozialen Rahmenbedingungen vorgenommen werden. Wenn das Abendessen die wichtigste familiäre gemeinsame Mahlzeit ist, scheint es wenig sinnvoll diese wegzulassen. $\mathrm{Zu}$ beachten ist, dass etwa $3 \mathrm{~h}$ vor dem Schlafengehen nicht mehr gegessen wird, parallel zum Anstieg der Melatoninsekretion. Bezüglich des Weglassens des Frühstücks gibt es weniges zu beachten. Kaffee und Tee ohne Milch und Zucker sind erlaubt. Viele Menschen haben auch instinktiv morgens wenig Hunger, was dann diese Form des Intervallfastens in natürlicher Weise begünstigt.

Als etwas extremere Form des TRE gilt die „Warrior diet“ bei der nur einmal am Tag, meist zwischen 17 und 20 Uhr, die gesamte tägliche Kalorienmenge verzehrt wird. Diese Form des Fastens ist besonders bei Sportlern beliebt.

Die bisher durchgeführten klinischen Studien zum TRE bestätigen eine sehr gute Wirksamkeit hinsichtlich Gewichtsabnahme und verbessertem Metabolismus [1]. Insbesondere bei Diabetes mellitus sind eindrückliche günstige Wirkungen für $16: 8$ beschrieben.

\section{Entlastungstage}

In der Naturheilkunde sind wöchentliche Entlastungstage seit langem etabliert. Hierbei wird angeraten, an einem Tag in der Woche nur Reis oder nicht zu süßes Obst mit einer Gesamtkalorienmenge von $1000 \mathrm{kcal} z u$ sich zu nehmen. Dies würde auch dem intermittierenden Fasten, im Sinne eines 1:6-Fastens, entsprechen. Allerdings konnten wir in einer eigenen unlängst publizierten Studie keine signifikanten Effekte durch alleinige wöchentliche Entastungstage in einer Pilotstudie bei gesunden normalgewichtigen Probanden feststellen [16]. Daher empfehlen wir derzeit Entlastungstage v.a. als sinnvolle Ergänzung zu einer pflanzenbasierten Ernährung nach einem Heilfasten.

Grundsätzlich können auch verschiedene Fastenformen miteinander kombiniert werden und erscheinen als durchaus sinnvolle Kombinationen, d.h., jährlich kann 1-2-mal ein längeres Heilfasten bzw. therapeutisches Fasten durchgeführt werden und im Alltag kann versucht werden, möglichst häufig ein intermittierendes Fasten bzw. Time restricted eating durchzuführen. Bewährt hat sich auch in der Verhaltensmedizin einen sog. „cheat day“ (Schummeltag) einzubauen, d.h., den Patienten zu erlauben bzw. anzuraten, an einem Tag der Woche keine feste Regel einzuhalten, um die Langzeit-Compliance zu erhöhen.

Während beim intermittierenden Fasten die Art der Nahrungszufuhr nicht weiter beeinflusst wird, zeigen neuere Daten der tierexperimentellen Forschung, dass ein Teil des Fasteneffekts grundsätzlich auch auf der Reduktion von tierischem Eiweiß und zugeführtem Zucker bzw. schnell resorbierbarem Kohlenhydraten beruht. Durch beide Restriktionen kann also bereits eine deutliche Absenkung der Konzentration von Insulin, IGF-1 sowie mTor induziert werden, die wesentlich für die Effekte des Fastens sind. Dies gab auch Valter Longo den Anlass, die Fasting mimicking diet zu entwickeln.

Insofern ist Patienten nach heutigem Kenntnisstand zu empfehlen, nach dem Fasten bzw. generell eine mehr pflanzenbasierte und zuckerarme Ernährung einzuhalten, um Synergieeffekte zu erzielen. Für die eigentliche Fastenphase legen diese neueren wissenschaftlichen Daten den Schluss nahe, die zugeführten Fastenspeisen möglichst frei von tierischem Eiweiß und raffiniertem Zucker zu halten. Daher sind vegane Fastenformen wie das Buchinger-Fasten von Vorteil. Bei der Auswahl der zugeführten Säfte sollte darauf geachtet werden, eher Gemüsesäfte statt süßer Obstsäfte zu verwenden.

\section{Fasten bei Krebs}

Eine Sonderrolle kommt dem Fasten bei Krebs zu. Eine Fülle von tierexperimentellen Daten weist auf die präventive, aber auch die therapeutische Wirkung von Kurzzeitfasten bei Krebserkrankungen hin. Andererseits ist Untergewicht mit einer ungünstigen Prognose bei Krebserkrankungen assoziiert, sodass es gilt, dieses unbedingt zu vermeiden. Klinisch am besten herausgearbeitet ist derzeit das Fasten über 72-84 Stunden begleitend zur Chemotherapie. In einer ersten Pilotstudie konnten wir hierbei signifikante Wirkungen auf Fatigue und Lebensqualität im Vergleich zu einer Normalkost feststellen. Derzeit untersuchen wir die Wirksamkeit des 72-Stunden-Fastens begleitend zur Chemotherapie in 2 größeren klinischen randomisierten Studien. Valter Longo und sein Team führen mehrere Studien zur Evaluation einer Fasting mimicking diet mit „Chemolieves“ während der Chemotherapie durch. Ergebnisse dieser Studi- en werden für 2018 erwartet, bis dahin können noch keine konkreten Empfehlungen ausgesprochen werden.

\section{Wie und warum wirkt Intervallfasten?}

Grundsätzlich scheint es naheliegend, dass der menschliche Körper in seiner evolutionären Entwicklung auf Phasen des Fastens bzw. Hungerns sehr gut eingestellt ist und diese gut bewältigen kann. Abwechselnde Phasen des kurzzeitigen Nahrungsüberangebotes und des Hungerns sind vermutlich der Regelfall des Frühmenschen gewesen. Entsprechend haben sich die genetische Ausstattung, der Körpermetabolismus und die Ernährungsphysiologie sehr gut darauf eingestellt. Im Gegensatz dazu ist anzunehmen, dass die derzeit häufig praktizierte regelmäßige Nahrungszufuhr von mehreren täglichen Mahlzeiten, in den letzten 20 Jahren auch zunehmend mit Zwischenmahlzeiten und Snacks sowie energiehaltigen Getränken, als unphysiologisch zu bewerten ist und den Körper in mannigfaltigen Stoffwechselvorgängen überfordert. Physiologischerseits stellt jede Essenszufuhr zunächst einen zellulären Stress dar, der für die Energieverarbeitung notwendig ist. Darüber hinaus werden der Verdauungsapparat und die energieverarbeitenden Hormon- und Stoffwechselsysteme stark aktiviert und bei dauerhafter Aktivierung durch pausenlose Nahrungszufuhr letztlich überlastet mit der Folge einer entsprechenden Rezeptorresistenz vieler Signal- und Steuerungssysteme.

Demgegenüber sind in vielen physiologischen Arbeiten die günstigen metabolischen Wirkungen des Fastens präzise beschrieben. Initial kommt es zunächst durch den Hunger zu einer Aktivierung der Stresshormone Adrenalin, Noradrenalin, Dopamin und etwas verzögert Cortisol. Zum anderen kommt es zu einer Leptin-Depletion und Erhöhung der Adiponektine mit entsprechend günstigen Wirkungen auf den Fett- und Zuckerhaushalt. Die leichte endokrine Stressantwort kann als günstig eingestuft werden, da sie im Sinne einer zellulären Adaptation (Hormesis) mit dazu beiträgt, dass weitere positive Wirkungen des Fastens induziert werden. $\mathrm{Zu}$ beachten ist dabei auch, dass es zellulär zu einer Abnahme der Glukokortikoid-Rezeptorexpression kommt.

Als Folge der fasteninduzierten Hormesis sind besonders herauszustellen:

1. Leistungssteigerung und Neubildung von Mitochondrien (Mitohormesis) 
2. Absenkung bzw. Normalisierung der häufig erhöhten Insulinspiegel, verbesserte Insulinsensitivität

3. Förderung der Stammzellenproduktion

4. Förderung der zellulären Reinigungsmechanismen via Autophagie

5. Neurobiologisch vermehrte zentrale Serotoninverfügbarkeit sowie eine Ausschüttung von Endorphinen und Endocannabinoiden (Erklärung für die häufig zu beobachtende gute Stimmung bis hin zur Fasteneuphorie während des Fastens)

Für die mutmaßlich protektive und therapeutische Wirkung des Fastens auf neurodegenerative Erkrankungen wie Multiple Sklerose und Demenz scheint v.a. die vermehrte Ketonbildung von Bedeutung zu sein.

Darüber hinaus führt psychologisch ein erfolgreiches Fasten zu einer deutlichen Stärkung der Selbstwirksamkeit und Eigenkompetenz.

Schließlich kann als weiterer gesundheitsfördernder Effekt des Fastens der geringe Anfall von Stoffwechsel- und Verdauungsarbeit für den Körper angesehen werden. Jede Energiezufuhr bedeutet für den Körper auf verschiedenen Ebenen einen physiologischen Stress. Dies beginnt mit der Verdauungstätigkeit im Darm, wobei jede Nahrungszufuhr auch die Auseinandersetzung mit einem Fremdkörper darstellt. Entsprechend werden die postprandiale Leukozytose bzw. nach neueren Arbeiten die Anhebung von NF-KB als Ausdruck dieser physiologischen Stressreaktion beschrieben. Auf mitochondrialer Ebene entstehen bei jeder Nahrungsaufnahme vermehrt Radikale. Eine kalorische Restriktion kontinuierlich oder intermittierend über Fasten führt dementsprechend zu einer geringeren Beanspruchung bzw. „Abnutzung“ der an der Energiegewinnung beteiligten Zellfunktionen.

Neuere Studien belegen zudem, dass verschiedene Formen des Fastens offensichtlich günstige Wirkungen auf das intestinale Mikrobiom ausüben. Erste Studien zeigen eine größere Diversität des Mikrobioms nach Heilfasten, aber auch durch Intervallfasten.

Für das Time restricted eating scheint grundsätzlich die damit induzierte bessere chronobiologische Rhythmisierung ein wichtiger günstiger Wirkmechanismus. Grundlagenwissenschaftler wie Satchidananda Panda vom Salk Institut in den USA verweisen darauf, dass durch viele tägliche Mahlzeiten ein dauerhafter metabolischer Jetlag im Körper entsteht, der durch Time restricted eating wieder überwunden werden kann.

Interessenkonflikt: Der Autor erklärt, dass keine wirtschaftlichen oder persönlichen Verbindungen bestehen.

\section{Online zu finden unter}

http://dx.doi.org/10.1055/a-0591-8783

\section{Literatur \\ 1 Patterson RE, Sears DD. Metabolic Effects of Intermittent Fasting. Annu Rev Nutr 2017; 37 : 371-393 \\ $\overline{2}$ Mattson MP, Longo VD, Harvie M. Impact of intermittent fasting on health and disease pro- cesses. Ageing Res Rev 2017; 39: 46-58 \\ $\overline{3}$ Longo VD, Panda S. Fasting, circadian rhythms, and time-restricted feeding in healthy lifespan. Cell Metab 2016; 23 (6): 1048-1059 \\ $\overline{4}$ Horne BD, Muhlestein JB, Anderson JL. Health effects of intermittent fasting: hormesis or harm? A systematic review. Am J Clin Nutr 2015; 102 (2): 464-470}

5 Wei M, Brandhorst S, Shelehchi M et al. Fastingmimicking diet and markers/ risk factors for aging, diabetes, cancer, and cardiovascular disease. Sci TransI Med 2017; 9 (377): doi: 10.1126/scitransImed.aai8700

$\overline{6}$ Michalsen A, Li C. Fasting therapy for treating and preventing disease - current state of evidence. Forsch Komplementmed 2013; 20 (6): 444-453

7 Mattson MP, Allison DB, Fontana L et al. Meal frequency and timing in health and disease. Proc Natl Acad Sci U S A 2014; 111 (47): 1664716653

$\overline{8}$ Longo VD, Mattson MP. Fasting: molecular mechanisms and clinical applications. Cell Metab 2014; 19 (2): 181-192

$\overline{9}$ López-Otín C, Galluzzi L, Freije JMP et al. Metabolic control of longevity. Cell 2016; 166 (4): 802-821

$\overline{10}$ Longo VD, Antebi A, Bartke A et al. Interventions to slow aging in humans: are we ready? Aging Cell 2015; 14 (4): 497-510

$\overline{11}$ www.L-nutra.com

$\overline{12}$ Harvie MN et al. The effects of intermittent or continuous energy restriction on weight loss and metabolic disease risk markers: a randomized trial in young overweight women. Int J Obes (Lond) 2011; 35: 714-727

$\overline{13}$ Moseley M. The fast diet. London: Randomhouse; 2013

$\overline{14}$ www.naturalrhythmeating.org $\overline{15}$ de Cabo R, Carmona-Gutierrez D, Madeo F et al. The search for antiaging interventions: from elixirs to fasting regimens. Cell 2014; 157 (7): 1515-1526

$\overline{16}$ Kessler CS, Stange R, Schlenkermann M et al. A nonrandomized controlled clinical pilot trial on 8 wk of intermittent fasting ( $24 \mathrm{~h} / \mathrm{wk})$. Nutrition 2018; 46: 143-152.e2

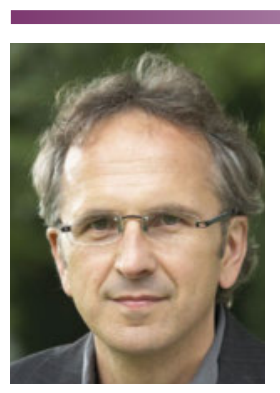

Prof. Dr. med. Andreas Michalsen

Stiftungsprofessur für klinische Naturheilkunde Charité - Universitätsmedizin Berlin

Abteilung Naturheilkunde

Immanuel Krankenhaus Berlin, Standort Berlin-

Wannsee

Königstraße 63

14109 Berlin

a.michalsen@immanuel.de

Andreas Michalsen ist Facharzt für Innere Medizin mit den Zusatzbezeichnungen Naturheilverfahren, Ernährungsmedizin, Physikalische Medizin und Balneologie. Nach Stationen in Berlin und Bad Elster war er von 1999-2008 leitender Oberarzt der Abteilung Innere Medizin V, Naturheilkunde und Integrative Medizin der Kliniken Essen-Mitte. Seit 2009 Inhaber der Professur für klinische Naturheilkunde, Charité - Universitätsmedizin und Immanuel Krankenhaus Berlin sowie Vorstandsvorsitzender der Carstens-Stiftung. 